$\xi=$

\title{
Digital Approaches in Industrial Design
}

\author{
K.S. Ivshin \\ Udmurt State University, Izhevsk, Russia
}

\begin{abstract}
In the present paper the digital approaches in industrial design have been developed and described, which reveals interdisciplinary connections for solving the problems of universal design, and searches for new forms of industrial products and investigates new types of industrial design of industrial products. The inversion modeling approach in stages resembles the traditional one, however, to create a surface model, the data obtained by scanning a man-made layout or prototype on a three-dimensional scanner are used. Shaping is based on the results of three-dimensional scanning, i.e. the source data is a point field or a polygonal model. Suitable for modeling a body approaching the final stage of production or for restyling the existing body shape. This approach is more laborious than the traditional one, due to the fact that you need to create a layout or have a ready prototype available, spend time on scanning, however, the maximum approximation of body parameters to a real object or layout is achieved. Criteria for choosing the inversion principle of modeling: according to TK, as a source for design data, a plasticine model, the form is not fractal, does not require changes in time and space, but is a complex closed shell.
\end{abstract}

Keywords: Digital, Industrial Design, 3D scanning, CNC Design

\section{Introduction}

For many years in the world practice of industrial design, a traditional design process has emerged for the development of a new industrial model. Design modeling in industrial design is already one of the main means of implementing the artistic concept of the form. Design practice is based on the constant updating of forms of industrial products, rethinking the interaction and structure formation of objects and space. Reducing timing of future product modeling is an urgent task. This task allows to solve the introduction of interdisciplinary and innovative approaches in the process of design-design of industrial products.

\section{Research method}

In industrial design, types of modeling are used in the synthesis [1-7], forming a certain principle for creating the shape of an industrial product. As a result of the analysis of the application of types of modeling [1-7], four modeling approaches were formulated and developed: 1) traditional, 2) inversion, 3) generative, 4) interactive. The basis of the traditional and inversion approaches is geometric modeling.

Traditional approach: 1) creative man-made (Sketch modeling) or polygonal modeling (Polygonal and mesh modeling); 2) surface modeling (Surface modeling); 3) solid modeling; 4) prototyping (Prototyping).

Inversion approach: 1) traditional (man-made) modeling (Modeling); 2) three-dimensional scanning (3D scanning); 3) surface modeling of class "A" / "B" (Surface modeling); 4) solid modeling; 5) prototyping (Prototyping).

In the traditional and inversion modeling approaches, the most important role is played by the stage of surface modeling. The basis of industrial design is high-quality surface modeling. The requirements for surface modeling are constantly increasing simultaneously with the cheapening of rapid prototyping technologies; conducting a reliable numerical analysis of strength, aerodynamics, etc. [7]; the use of system design (where one model simultaneously goes to the creation of advertising, exhibitions, production). These tasks are currently being solved using high-quality surface models.

Composite surface modeling. Differentiation of polystyrene surfaces. Any model consists of many different elementary surfaces, which leads to the need to dock different surfaces by origin with each other. Achieving a visually indistinguishable joint of a matte or semi-matte surface is achieved in class "B"; however, in the presence of a glossy surface of a visually indistinguishable joint and a smooth flare transition, the construction of class a surfaces is required.

Surface analysis. To illustrate and analyze how real surfaces will look, various ways of analyzing curves and surfaces are used. However, the most informative for identifying and analyzing surfaces of class "A", "B" and "C" are "Curve curvature diagram" ("Curve curvature") and "Isophot analysis" ("Isophot analysis").

The analysis is based on an estimate of the radii of curvature. The curvature plot is characterized by perpendicular to the tangents on the analyzed curve, straight lines (the number of which can be specified manually) and height of the given transverse straight lines, which reflect the curvature of the surface and reveal the presence of curvature radii or their absence. This type of analysis of curves and edges of surfaces is based on the identification of irregularities. The curvature diagram increases the camber, valleys and curves of the curve. It also shows the curve of the curve. The dialog box allows you to change the size and density of the "cross lines". The possibility of analysis is the identification and graphical representation of the maximum and minimum radius of curvature.

Isophot analysis appeared before the advent of computers and was based on the illumination of an object through the surface, cut by long equidistant stripes through which light evenly penetrated, 
forming isophotes. Isophot analysis simulates reflex lines. This type of analysis gives a complete picture of the distribution and shape of glare on the surface of a simulated object, and allows you to visually distinguish class a surfaces with high accuracy.

Generative approach: 1) information modeling (Information modeling); 2) geometric modeling (Geometry modeling); 3) prototyping (Prototyping). Generative modeling is gaining great popularity among designers, which today is widely used in parametric and generative shaping of objects [8-10].

Interactive approach: 1) Generative modeling; 2) decoding of the information model in the "Firefly" plugin (Decoding); 3) an open platform for prototyping "Arduino" / "Freduino" (Interactive prototyping).

\section{Results and analysis}

The traditional approach provides a simulation of concept art concepts and final forms of the vehicle body with different complexity of geometry. The criteria for choosing the traditional principle of modeling: shell structure of the body shape, not fractal, does not require changes in time and space; The initial data for the simulation are sketches and drawings.

The inversion modeling approach in stages resembles the traditional one, however, to create a surface model, the data obtained by scanning a man-made layout or prototype on a threedimensional scanner are used. Shaping is based on the results of three-dimensional scanning, i.e. the source data is a point field or a polygonal model. Suitable for modeling a body approaching the final stage of production or for restyling the existing body shape. This approach is more laborious than the traditional one, due to the fact that you need to create a layout or have a ready prototype available, spend time on scanning, however, the maximum approximation of body parameters to a real object or layout is achieved. Criteria for choosing the inversion principle of modeling: according to TK, as a source for design data, a plasticine model, the form is not fractal, does not require changes in time and space, but is a complex closed shell.

The urgency of the generative approach arises when it is necessary to change the parameters of the objective system in space and time, or in the presence of a system of objects with a complex multipart non-repeating structure. The application of the interactive principle in industrial design is currently experimental [8-10]. Product: a form with a complex structure, a member of the sectors, patterns, fractals, etc.

The interactive principle is based on the integration of a generative model with an open platform for prototyping, by decoding the information model (the "Firefly" plugin). Such platforms allow you to create electronic systems that include sensors (light sensor, position in space, etc.) to interact with the environment, servos, motors, and more [11]. This method of modeling allows design students and practicing designers, as well as researchers, to create generative models that can interact with the environment with a person through sensors [12-17]. It is possible to create kinetic prototypes that change in real time as the generative model changes on the computer. At the moment, to create a prototype, students need to have basic knowledge of working with electrical circuits to connect sensors to a microprocessor. However, this problem is solved by the presence in the network of a large number of stepby-step lessons and a rather simple principle of application developed by Arduino.

\section{Conclusion}

In the present paper, digital approaches in industrial design are described, which reveal interdisciplinary communication for solving problems of universal design, searching for new forms of industrial products and researching new types of design modeling of industrial products. These approaches make it possible to intelli- gently choose a rational modeling approach in a specific project situation, look for new ways to embody an artistic vision, develop a new thinking and innovative approach to modeling industrial products.

\section{References}

[1] Russo M. Polygonal Modeling: Basic and Advanced Techniques (Wordware Game and Graphics Library), 2005.

[2] Lombard M. Solid Works 2011 Bible.

[3] Krüger R. Three Dimensional Finite Element Analysis of Multidirectional Composite DCB, SLB and ENF Specimens / R. Krüger // ISD-Report 1994. No.2

[4] Khabazi M. Algorithmic modeling with Grasshopper, 2009, [online] Available at: http://proquest.safaribooksonline.com/1587050773 [accessed 01/03/2012].

[5] Ivshin, K.S. High-quality surface modeling in the design of vehicles // Design. Theory and practice (electronic journal). - 2011. - № 7. p. 83-93.

[6] Ivshin, K.S. Electronic geometric modeling in the design of industrial products and vehicles // Design. Materials Technology. - 2009. - № 1. - C. 105-108.

[7] Ivshin, K.S. Numerical analysis in the design of small vehicles // Design. Theory and practice (electronic journal). - 2011. - № 8. - p. $1-14$.

[8] Shumacher P. Parametricism as Style - Parametricist Manifesto, 2008, [online] Available at: http://www.patrikschumacher.com/Texts/ Parametric-ism\% 20as\% 20Style.htm [accessed 01/03/2012].

[9] Brakke K. Triply Periodic Minimal Surfaces, 2000, [online] Available at: http: //www.susqu/edu / brakke / evolver / examples / periodic / periodic.html [accessed 01/03/2012].

[10] Geenen B. Gaudi Stool [online] Available at: http://www.studiogeenen.com/projects/ [accessed 01/03/2012].

[11] Johnson J.K., Payne A. Firefly Primer - version 1.006, 2011, [online] Available at: http://www.fireflyexperiments.com [accessed 01/03/2012].

[12] Zaha Hadid Arcitects [online] Available at: http://www.zahahadid.com [accessed 01/03/2012]. 11. Strelka. Institute for media, architecture and design. [online] Available at: http: // www. strelkainstitute.com [accessed 01/03/2012].

[13] Strelka. Institute for media, architecture and design. [online] Available at: http: // www. strelkainstitute.com [accessed 01/03/2012].

[14] Johnson J.K., Payne A. Firefl y Primer - version 1.006, 2011, [online] Available at: http: // www.fi refl yexperiments.com [accessed 01/03/2012].

[15] Zalek Y. Another Wave in the Wall: Vertical Lake Building Façade, 2010, [online] Available at: http://weburbanist.com/2010/07/23/another-wave-in-the-wallvertical- lake ... [accessed 01/03/2012].

[16] Elsacker E., Bontinckx Y. Kinetic pavilion, 2011, http://www.kineticpavilion.com [accessed 01/03/2012].

[17] Branchpoint project, 2010. [online] Available at: http://branchpoint.ru [accessed 01/03/2012]. 